\title{
Proctitis as the clinical presentation of lymphogranuloma venereum, a re-emerging disease in developed countries
}

\author{
Jorge López-Vicente, Daniel Rodríguez-Alcalde, Luis Hernández-Villalba1, Diego Moreno-Sánchez¹, \\ Mercedes Lumbreras-Cabrera ${ }^{1}$, Carlos Barros-Aguado ${ }^{2}$ and Juan Carlos Galán ${ }^{3}$ \\ Departments of ${ }^{1}$ Gastroenterology and ${ }^{2}$ Infectious Diseases. Hospital Universitario de Móstoles. Madrid, Spain. \\ ${ }^{3}$ Department of Microbiology. Hospital Universitario Ramón y Cajal. Madrid, Spain
}

\begin{abstract}
Lymphogranuloma venereum (LGV) is a sexually transmitted infectious disease caused by serovars L1, L2 and L3 of Chlamydia trachomatis. The initial presentation is usually a painless ulcerated papule on the genitalia or distal proctitis. The progression of the infection can lead to major complications: rectal strictures, intestinal obstruction or perforation. We present five cases of LGV proctitis as the initial presentation of the disease. All patients were male, mean age 44.6 years, with positive serology to human immunodeficiency virus (HIV) and promiscuous men who have sex with men (MSM). The initial diagnosis was made by rectosigmoidoscopy indicated for pain and anal discharge. All cases were confirmed by polymerase chain reaction technique in rectal tissue. Endoscopic images obtained showed a great variety of rectal lesions, from mild erythema of the mucosa and ulcers to deep ulcers with elevated borders and purulent exudate. All cases were resolved after treatment with doxycycline for 3 weeks. It emphasizes the importance of suspecting this re-emerging disease in patients with risk factors (HIV and MSM), with the aim of early treatment and to avoid major complications.
\end{abstract}

Key words: Proctitis. Chlamydia. Lymphogranuloma venereum. Men who have sex with men. Human immunodeficiency virus.

López-Vicente J, Rodríguez-Alcalde D, Hernández-Villalba L, Moreno-Sánchez D, Lumbreras-Cabrera M, Barros-Aguado C, Galán JC. Proctitis as the clinical presentation of lymphogranuloma venereum, a re-emerging disease in developed countries. Rev Esp Enferm Dig 2014;106:59-62.

Received: $15-07-2013$

Accepted: $19-08-2013$

Correspondence: Jorge López Vicente. Department of Gastroenterology. Hospital Universitario de Móstoles. c/ Río Júcar, s/n. 28935 Móstoles, Madrid. Spain

e-mail: lopezvicentej@ hotmail.com

\section{INTRODUCTION}

Lymphogranuloma venereum (LGV) is a sexually transmitted infectious disease caused by serovars L1, L2 and L3 of Chlamydia trachomatis. Before 2003, it was considered a rare disease in industrialised countries but it was endemic in parts of Africa, Asia, South America, and Caribbean countries. The first European case was detected in Rotterdam, the Netherlands, in February 2003 and since then there have been clusters of cases in European countries, North America and Australia. The first case in Spain was reported in 2005 in Barcelona (1).

The clinical course of the disease includes three stages. First, after an incubation period of 3 to 30 days, it is characterized by the appearance of small painless ulcerated papule in the site of inoculation (prepuce or glans in males and vulva or vaginal wall in women). This lesion is selflimiting and may pass unnoticed by most patients. In the second stage which begins a few weeks or even months after the primary lesion, loco-regional lymphatic chains in the site of inoculation are affected and painful inguinal lymphadenopathy usually appears. Anal ulcers or proctitis may also arise by direct inoculation of the bacteria in the rectal mucosa, a more common presentation in women or men who have sex with men (MSM). These patients report anal pain and/or rectal bleeding often accompanied by fever or chills. At rectosigmoidoscopy several lesions can be seen in distal rectum, from a nodular mucosal erythema to ulcers with mucopurulent exudate. In the third stage, which occurs when the disease progresses untreated, lymph node necroinflammation leads to obstruction of lymphatic drainage and genital elephantiasis. In this context, rectal involvement may be complicated by fistulae and rectal strictures (2).

The current standard method for diagnosing LGV is the direct identification of Chlamydia trachomatis in tissue or fluids from the patient. Initially laboratory diagnosis was based on cultures, today used only in specific cases, or in the nucleic acid amplification test followed by genotyp- 
ing with polymerase chain reaction (PCR). Since 2005, molecular biological techniques based on high specificity detection of the membrane protein $\mathrm{H}$ gene by PCR are used (3). Serology has also been replaced by direct detection tests, but still may have some utility in selected cases (e.g. in patients with symptoms when samples or PCR techniques are not available), as high titers of serum antibodies is very suggestive of LGV.

The treatment of choice is doxycycline $100 \mathrm{mg}$ orally twice daily for 3 weeks. Other alternatives are erythromycin $500 \mathrm{mg}$ orally four times daily for 3 weeks or a single oral dose of azithromycin $1 \mathrm{~g}$ once weekly for 3 weeks. Response to antibiotic treatment is usually complete after 3 or 6 weeks and all lesions disappear in most patients. In case of complicated disease with fistulas or anal strictures, and after antibiotic treatment, surgery or aspiration and drainage of lymph nodes may be needed. Up to now, and in our knowledge, there has been no reported case of endoscopic dilation in anal strictures, although it could be an alternative procedure to consider.

\section{CASE REPORTS}

We report 5 cases of LGV in white men with proctitis as the initial presentation lesion who were diagnosed between February 2011 and February 2012. The mean age was 44.6 years and all cases were seropositive for human immunodeficiency virus (HIV). Four were receiving antiretroviral treatment and had undetectable viral load at the time of infection with Chlamydia trachomatis. The other case was diagnosed with HIV infection in this episode (Table I). All of them were MSM and had multiple sexual partners in the last months. The symptoms that led to the request of a rectosigmoidoscopy were anal pain in three of them and anal rectal discharge associated with rectal bleeding in the others. Endoscopic involvement was limited to the

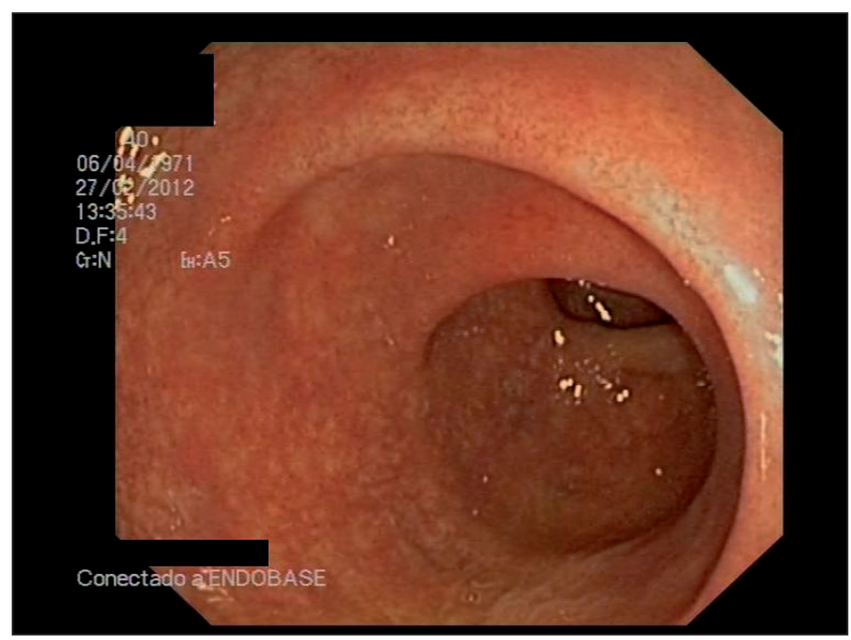

Fig. 1. Mild mucosal erythema in rectum (case 5).

distal rectum in the first three cases and to the middle and distal rectum in the last two. The lesions ranged from mild and nonspecific hyperemic mucosa (case 5, figure 1) to a circumferential ulceration with elevated geographical borders and mucopurulent exudate (case 3; figures 2 and 3). Pathological examination of rectal biopsies showed granulation tissue, increase in polymorphonuclear leucocytes, fibrin exudate associated with fibrosis and stromal congestion. There were no granulomas or viral inclusions in the samples. Periodic acid-Schiff and Ziehl-Nielsen stains were negative. All cases were confirmed with PCR for L serotypes of Chlamydia trachomatis in rectal tissue. Case 3 showed inguinal lymphadenopathy in pelvic computed tomography. All patients were treated with doxycycline, $100 \mathrm{mg}$ orally twice daily for 3 weeks, with complete resolution of all lesions. Mucosal healing was confirmed by sigmoidoscopy within 2 to 4 weeks after the treatment. In both cases with more severe mucosal injury (cases 3 and 4 )

Table I. Patients characteristics with LGV proctitis

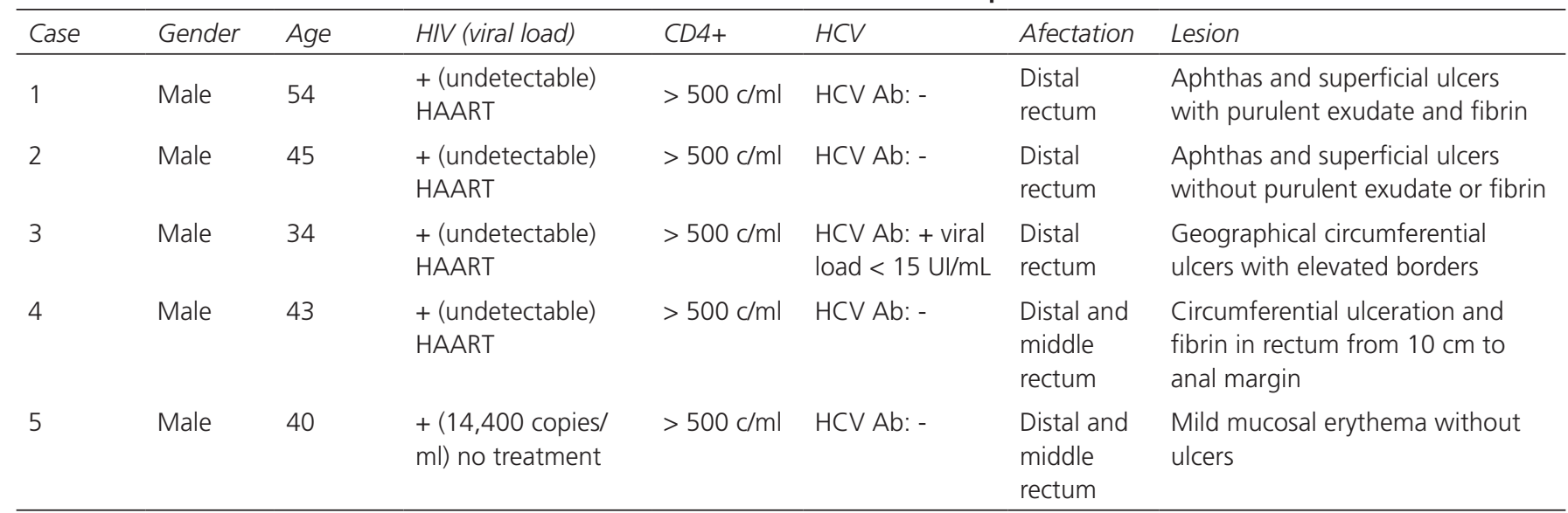

HIV: Human immunodeficiency virus; HCV Ab: Hepatitis C virus antibodies; HAART: Antiretroviral therapy. 


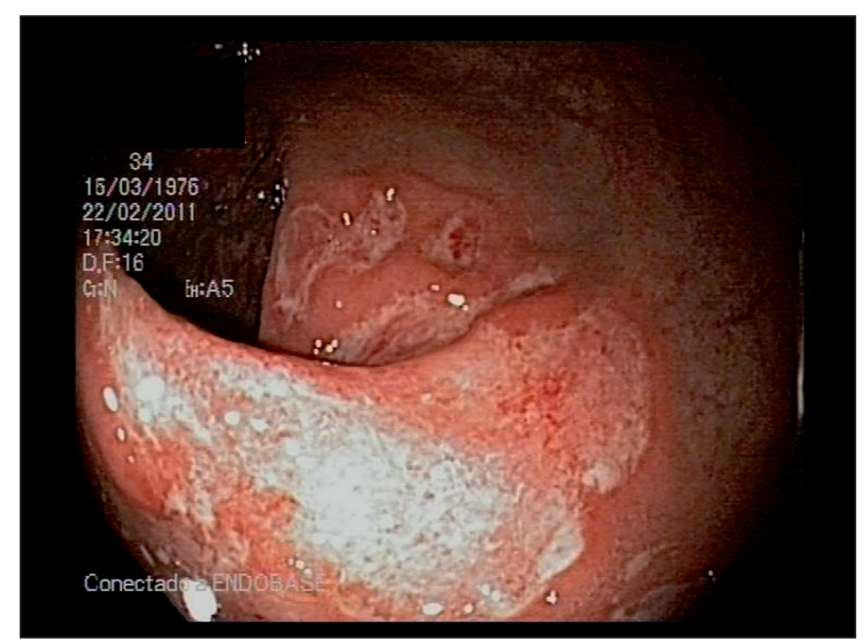

Fig. 2. Geographical ulcers in rectum (case 3).

distal rectal scarring remained, without stenosis or other complications. After a mean follow up of 15.2 months, all patients were asymptomatic without rectal lesions.

\section{DISCUSSION}

There has been a resurgence of $\mathrm{LGV}$ in the last ten years in urban areas of developed countries, particularly among MSM. Recent publications show a significant prevalence of co-infection with HIV (up to $70 \%$ in European series) (4) and a high proportion of these cases are promiscuous MSM. It has been observed also a co-infection with other sexually transmitted diseases such as syphilis, gonorrhea or hepatitis $C$ virus (5). It seems that these individuals do not represent a reservoir of the disease. The co-existence of both infections is not only the result of high sexual risk behavior but it could be a synergistic effect between HIV and LGV (6). Furthermore, it is not considered an opportunistic infection as most of the cases described were under antiretroviral treatment and with normal CD4 lymphocyte count. Some authors suggest that the increase of cases reported in recent decades is due to the development of diagnostic tests for serotypes L1, L2 and L3 of Chlamydia since 2003, which have emerged these infections previously undiagnosed. Other authors speculate that a change in antibiotics prescribing practices for urethritis and other genital infections in the 90's, with a decrease in the use of doxycycline and an increase in azithromycin, would have re-emerged Chlamydia endemic infections (7).

In the reappearance of this disease its presentation has changed and, today, proctitis is the most common initial clinical manifestation. Thus, in over $90 \%$ of the cases reported in France, the UK and the Netherlands, rectal discomfort and proctitis were the initial complaint of the patients (8-10). Then differential diagnosis of LGV includes other sexually transmitted diseases such as syphi-

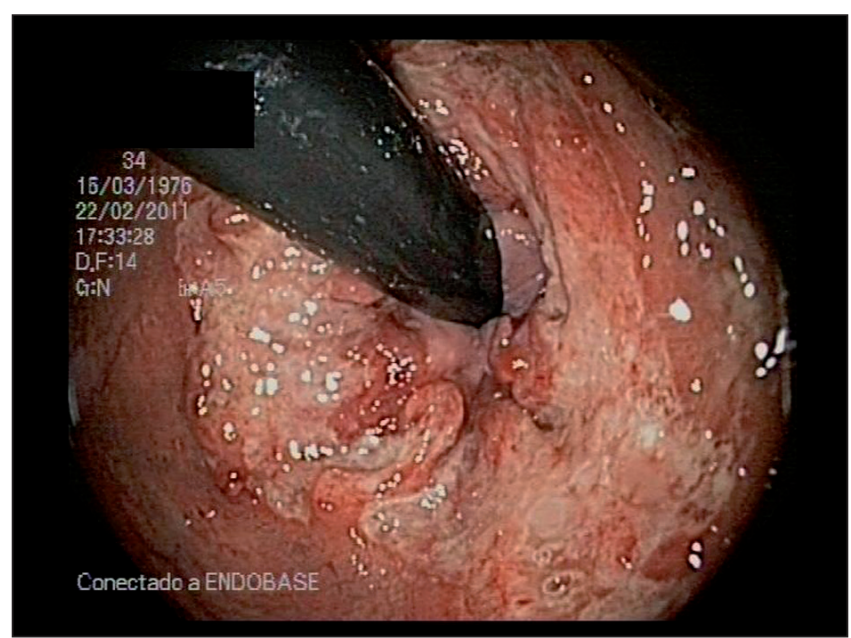

Fig. 3. Distal rectum mucosa with a $100 \%$ circumferential ulceration and fibrin, in retroflexed view (case 3 ).

lis, gonorrhea or proctitis caused by herpes simplex virus or cytomegalovirus. In severe rectal lesions, endoscopic images can mimic an inflammatory bowel disease (11), a rectal carcinoma or a lymphoma.

In this series of five cases, all patients were promiscuous MSM. Despite HIV infection there was no immune deficiency as in all patients CD4 lymphocytes count was in normal range. The route of inoculation of the microorganism was the anal sexual activity, but the presenting symptoms ranged from mild discomfort to rectal anal suppuration and rectal bleeding, perhaps related to the severity of proctitis or to the time from infection. Also notable is the variety of rectal mucosal lesions induced by Chlamydia trachomatis and that are showed in the pictures. Two of the patients had full circumference rectal ulcers and mucopurulent exudate that mimicked anal carcinoma or Crohn's disease, particularly when those lesions were detected on the digital rectal exploration. By contrast, in two other patients, one of whom was diagnosed of HIV infection in that episode, the rectal findings were minimal showing mild mucosa erythema and some aphthas, so the infection could be passed unnoticed.

LGV is a public health problem. Case detection and study of contacts to prevent the spread of the disease is a challenge for all health professionals. It is necessary to define the diagnostic targets and the monitoring of risk populations to prevent the increase in cases and the concomitant infections. Gastroenterologists are beholden to suspect this emerging disease when compatible symptoms are present, especially in MSM, and not ignore an endoscopic examination and mistaken this disease with trivial anal problems. The endoscopist, due to the large spectrum of possible rectal lesions, should take biopsies routinely for LGV and other sexual transmitted disease and request the laboratory for PCR in tissue of Chlamydia trachomatis serovars $\mathrm{L}$ when suspecting this disease. 


\section{REFERENCES}

1. Martin-Iguacel R, Llibre JM, Nielsen H, Heras E, Matas L, Lugo R, et al. Lymphogranuloma venereum proctocolitis: A silent endemic disease in men who have sex with men in industrialized countries. Eur J Clin Microbiol Infect Dis 2010;29:917-25.

2. Mabey D, Peeling RW. Lymphogranuloma venereum. Sex Transm Infect 2002;78:90-2.

3. Morré SA, Ouburg S, van Agtmael MA, de Vries HJ. Lymphogranuloma venereum diagnostics: From culture to realtime quadriplex polymerase chain reaction. Sex Transm Infect 2008;84:252-3.

4. Van de Laar MJW. The emergence of LGV in Western Europe: What do we know, what can we do? Euro Surveill 2006; 11: 146-8.

5. Götz HM, van Doornum G, Niesters HG, den Hollander JG, Thio HB, de Zwart $\mathrm{O}$. A cluster of acute hepatitis $\mathrm{C}$ virus infection among men who have sex with men: Results from contact tracing and public health implications. AIDS 2005;19:969-74.
6. Rönn MM, Ward H. The association between Lymphogranuloma venereum and HIV among men who have sex with men: Systematic review and meta-analysis BMC Infectious Diseases 2011;11:70.

7. Schachter J, Moncada J. Lymphogranuloma venereum: How to turn an endemic disease into an outbreak of a new disease? Start looking. Sex Transm Dis 2005;32:331-2.

8. Jebbari H, Alexander S, Ward H, Evans B, Solomou M, Thornton A, et al. Update on lymphogranuloma venereum in the United Kingdom. Sex Transm Inf 2007;83:324-6.

9. Herida M, de Barbeyrac B, Sednaoui P, Scieux C, Lemarchand N, Kreplak G, et al. Rectal lymphogranuloma venereum surveillance in France 2004-2005. Euro Surveill 2006;11:155-6.

10. Ward H, Martin I, Macdonald N, Alexander S, Simms I, Fenton K, et al. Lymphogranuloma venereum in the United kingdom. Clin infect Dis 2007; 44: 26-32.

11. Gallegos M, Bradly D, Jakate S, Keshavarzian A. Lymphogranuloma venereum proctosigmoiditis is a mimicker of inflammatory bowel disease. World J Gastroenterol 2012;18:3317-21. 